\title{
Mikro- und Makroperspektiven in der Entwicklungsdiskussion
}

\author{
Von Wolfgang Spoerr
}

\section{Karin Stahl}

Kuba - eine neue Klassengesellschaft?

Heidelberg 1987 (Reihe Heidelberger Dritte Welt Studien, Band 23), DM 36,-

\section{Heinrich Kreft}

Entwicklung durch Partizipation. Möglichkeiten der Interessendurchsetzung marginalisierter Gruppen in Entwicklungsländern, analysiert am Beispiel der "Unión de Organizaciones Campesinas de Rio de Oro" in Ecuador.

Lit Verlag, Münster 1987

Dieter Nohlen / Peter Waldmann (Hrsg.)

Dritte Welt. Gesellschaft - Kultur - Entwicklung.

Band 6 von Pipers Wörterbuch zur Politik, München 1987, DM 86,-

\section{Manfred Wöhlcke}

Der Fall Lateinamerika. Die Kosten des Fortschritts.

Beck'sche Reihe 394, München 1989, DM 17,80

In ihrer aus einer Heidelberger Dissertation entstandenen Studie "Kuba - eine neue Klassengesellschaft" legt Karin Stahl eine gründliche und kenntnisreiche Analyse der Gesellschaft der Karibikinsel vor. Normative Meßlatte ist ihr dabei ein System, das die "Verfügungsgewalt der unmittelbaren Produzenten über die verstaatlichten Produktionsmittel und das erzeugte Mehrprodukt (15 f.) und so die Mitwirkung der Subjekte an politischen und ökonomischen Entscheidungsprozessen gewährleistet. Damit sollen dann Entfremdung und Klassenstrukturen aufgehoben sein. Auf dieser sozialistischen, der "kritischen Theorie" verhafteten Basis kritisiert sie den Weg der kubanischen Revolution systemimmanent.

Da die sozialistische Weltanschauung auch die der herrschenden Gruppe in Kuba ist, interessieren die Ergebnisse von Stahl besonders. Hier wird die kubanische Revolution an ihren eigenen Ansprüchen gemessen. Das Ergebnis: In Kuba ist das "Kapital als Kapital verstaatlicht" worden (17), ohne daß die unmittelbaren Produzenten irgendwelche Verfügungsgewalt daran besäßen. "Die Produktionsverhältnisse bleiben Klassenverhältnisse" (17).

Im Gegensatz zu weiten Teilen der früheren Literatur zu Kuba lehnt Stahl eine Erklärung des kubanischen Weges mit externen Faktoren (abhängige Entwicklung, Druck der USA, 
Einfluß der Sowjetunion) ab (z.B. S. 21-22). Vielmehr sieht sie von ihr als "historischstrukturell" (22) bezeichnete interne Entwicklungen als dominant an.

Einer dichten, sehr lesenswerten Darstellung der Ausgangsbedingungen im vorrevolutionären Kuba (25-57) folgt eine ähnlich komprimierte Geschichte der Guerilla, die nicht ohne Unterstützung aus den Reihen der alten Eliten (z.B. der Armee, 73) vorwiegend vom städtischen Kleinbürgertum (76 f., 82-85) getragen wurde. Die städtische Industriearbeiterschaft hingegen schlo $\beta$ sich erst "in letzer Minute" $(88,104)$ an. Apologetisch behandelt wird denn auch die "Säuberung und Reorganisierung", mit anderen Worten: die Gleichschaltung und Unterdrückung der Gewerkschaften im nachrevolutionären Kuba als Mittel gegen den sogenannten "Ökonomismus" der Arbeiteropposition (102).

Mit der ersten großen Krise von 1970, die darauf zurückging, daß selbstgesetzte wirtschaftliche Ziele nicht erreicht wurden, geriet das System in eine interne Legitimitätskrise. In deren Folge wurde das wirtschaftliche Modell modifiziert, indem man das sowjetische System der "wirtschaftlichen Rechnungsführung" eingeführt hat. Den Details der Kommandowirtschaft osteuropäischen Musters wendet die Autorin viel Aufmerksamkeit zu (185-237), wobei sie es stets bei einer an dessen eigenen theoretischen Prämissen orientierten Kritik beläßt.

Der Aufbau des Staatsapparates wird - durchaus konsequent im Rahmen einer marxistischen Theorie - erst anschließend dargestellt (234-297). Erst nach einer Darstellung der technischen Einzelheiten des Wahlsystems (272-282) räumt die Verfasserin ein, daß die Wahlen, "gemessen an westlichen Demokratievorstellungen, keinen Austausch der ... politischen Führung ermöglichen", was aber "im Rahmen des staatlich gelenkten sozialen und ökonomischen Entwicklungsweges auch nicht intendiert" sei - so einfach ist das für die bei einer Forschungseinnichtung der evangelischen Kirche tätige Autorin.

Das Scheitem der kubanischen Revolution läge denn auch in der "Herausbildung neuer Klassenantagonismen und staatskapitalistischer Strukturen" (308). Dafür werden natürlich nicht etwa Mängel der Theorie selbst verantwortlich gemacht, vielmehr seien die "spezifischen Strukturen des abhängigen Kapitalismus" des Pudels Kern. Sie hätten sowohl die vollständige Herausbildung einer nationalen Bourgeoisie als auch eines homogenen Proletariats blockiert; ohne diese Klassen aber sei eine sozialistische Entwicklung nicht möglich. Obwohl Stahl den Anspruch erhebt, die Mikroebene der kubanischen Gesellschaft einzubeziehen (22), wird dies nicht eingelöst. Das mag aber auch damit zusammenhängen, daß sie das Land nur kurz und als Pauschaltouristin besuchen durfte. Ganz unwillkürlich fragt man sich bei der Lektüre des Buches, wieviele sozialistische Experimente eigentlich noch scheitern und zu unermeßlichem Leid und Elend führen müssen, bis die westliche und insbesondere die bundesdeutsche Linke endlich einmal ihre Theorien in Frage stellt und einsieht, daß es zur offenen Gesellschaft bürgerlicher, freiheitlicher Demokratien keine Alternativen gibt.

Wie wertvoll es sein kann, ausführlich die "Mikroebene" politischen Verhaltens zu untersuchen, also bei Einstellungen, Verhaltensmustern und Entwicklungen einzelner und sozialer 
Kleingruppen anzusetzen, zeigt das Buch von Heinrich Kreft über "Entwicklung durch Partizipation" in Ecuador. Einer konzisen und angenehm kurzen Einführung in die Problematik anhand der Punkte: Situation der Entwicklungsländer (3-7), soziale und wirtschaftliche Lage der armen Bevölkerung (7-11), öffentliche Verwaltung im Entwicklungsprozeß (14-18) sowie Partizipation der Marginalisierten (19-30) folgt eine ebenso lesenswerte Darstellung des Zusammenhangs zwischen Entwicklung, Partizipation und Organisation (31-63). Organisation wird als Vorbedingung für Partizipation dargestellt (39 f.). Im Gegensatz zwischen Genossenschaften nach westlichem Muster und autochthonen Selbsthilfe-Organisationen (SHO) gibt der Autor eindeutig letzteren den Vorzug (62). Die insbesondere in Deutschland nicht besonders umfangreiche Literatur zum Thema wird nachgewiesen, aber - zu Recht - nicht ausführlich dargestellt.

Das Untersuchungsgebiet sind die innerhalb des peripheren Landes Ecuador (dazu 64-121) Marginalisierten, also gewissermaßen die Peripherie der Peripherie, sowohl geografisch (131, 148 f.) als auch sozial (137). Der empirische Teil beruht auf einem Forschungsaufenthalt in der Region und einer umfangreichen Fragebogenaktion unter den betroffenen Campesinos.

Die Ergebnisse sind überaus interessant, vor allem als Darstellung der tagtäglichen Probleme der armen Landbevölkerung und ihrer Perzeptionen. Überragend ist die Bedeutung, die sie einer Verbesserung der Verkehrsinfrastruktur beimessen $(142,171,189,202$, 204, 211, 220, 255). Ihr Vertrauen in ihr Interessendurchsetzungs-Potential und in ihre SHO ist hoch (234, $247 \mathrm{f}$., 275); dem steht aber auch einiges an Resignation gegenüber, soweit es um die Möglichkeit gesamtgesellschaftlicher Verbesserungen geht (223 f.). Die objektiv besonders drückende Ausbeutung durch Zwischenhändler und Kredithaie etwa wird von den Leidragenden eher unterschätzt (98 f., 173, 185, 190), Lösungsansätze werden teilweise unsolidarisch konterkariert (174, $218 \mathrm{f}$.).

Den Erfolgen ihrer Organisation (186, 187, 181, 174, 269 ff.) steht eine zum Teil wohl bedenkliche Anspruchsmentalität gegenüber Entwicklungshilfemaßnahmen gegenüber (191, 260, 262, 264, 288), die diese vor allem als Leistungsgewährung erscheinen läßt. Wirtschaftliche Probleme werden vorwiegend als individuelle perzipiert (217).

Der Autor weist nach, daß die Unterstützung durch Entwicklungshilfe im Rahmen einer Selbsthilfe-Förderungsorganisation (SHF) entscheidend zum Erfolg der SHO beigetragen hat $(202,218,290 \mathrm{f} ., 301)$. Gegenüber herkömmlicher, auf den Verwaltungsapparat der Empfängerländer setzender Entwicklungshilfe erscheint diese Förderung als sehr viel effizienter. Dies wird empirisch belegt (205 f., 207, 215, 233, 257 f., 266, 292 f.), auch anhand der Auffassungen der Betroffenen. Auch von ihr dürfen aber keine Wunder erwartet werden; auch sie ist das geduldige, langsame Bohren dicker Bretter.

Das als Band 6 von Pipers Wörterbuch zur Politik herausgegebene Nachschlagewerk "Dritte Welt" zeichnet sich besonders dadurch aus, daß es eine einseitige Festlegung auf "Außen"-Aspekte vermeidet, insbesondere der allgemeinen Entwicklungstheorie, wie sie durch den Paradigmen-Gegensatz Modemisierung/Dependencia verkörpert wurde. Die 
insgesamt 70 Artikel führen gerade auch in interne, nicht ohne weiteres am Gegensatz Entwicklung/Unterentwicklung festzumachende Stichworte ein. Beispiele hierfür sind "Händler und Geldverleiher", "Familie", "Frauen", "Kirchen", "Gewerkschaften", "Rechtssysteme", "Politische Literatur", "Politische Kunst in Lateinamerika", "Politisches Lied", "Religiöse Bewegungen" und "Studenten".

Aber auch zu zentralen Konzepten der Entwicklungsdiskussion fehlen Einführungen nicht; besonders gelungen beispielsweise "Wohnverhältnisse/Habitat" und "Bevölkerung". Während nahezu alle Verfasser bewußt aus dem Kreis der deutschen Forschung gewählt wurden, sind die oft umfassenden Literaturangaben zu jedem Beitrag wertvolle Bibliographie auch zur internationalen Forschung. Einigen fast etwas knappen Artikeln ("Arbeit und Beschäftigung", "Menschenrechte") stehen ausgesprochen umfassende, dichte und weit über das Normale eines Lexikonartikels hinaus bedeutende gegenüber ("Demokratie/ Demokratische Systeme", "Autoritäre Systeme", "Infrastruktur"). Einige Themen werden regionenspezifisch behandelt ("Außenpolitik", "Familie", "Frauen", "Gewerkschaften", "Kirchen", "Mittelschichten", "Politische Kulturen", "Politische Parteien", "Präkoloniale Gesellschaften", "Soziale Sicherheit", "Sozialstrukturen", "Unternehmer", "Verwaltung"). Dies wäre zwar auch anderen Themen angemessen gewesen, hätte den Anspruch auf gebietsübergreifende Generalisierung aber in Zweifel gezogen.

Spezifisch entwicklungstheoretische Aspekte werden in zahlreichen Stichworten angesprochen. Unter dem Stichwort "Entwicklungstheorien" wird das Scheitem der Dependencia zwar eingeräumt, ihr aber dennoch eine "wichtige heuristische Funktion" (167) zugemessen. Für eine ausführliche Diskussion der einzelnen Denkrichtungen wird auf die Literatur und insbesondere auf das von Dieter Nohlen und Franz Nuscheler herausgegebene "Handbuch der Dritten Welt" verwiesen. Dagegen ist die Nachbardisziplin Entwicklungsökonomie mit empirisch fundierten Beiträgen zu "Wirtschaftspolitik", "Verschuldung" und "Emährung" vertreten.

Dem Anspruch, ein repräsentatives Kompendium des Standes der bundesdeutschen Drittwelt-Forschung zu schaffen, entspricht das Werk vollauf. Die breite Auswahl der Autoren sichert ein auch politisch pluralistisches Ergebnis; die große Mehrheit vertritt dabei Positionen im politikwissenschaftlichen "mainstream" zentristischer oder gemäßigt linker Positionen. Alles in allem ist Band 6 ein sehr gelungener Abschluß der Reihe, die weit mehr ist als nur ein Wörterbuch, nämlich ein umfassendes Kompendium über den Forschungsstand einer inzwischen wohletablierten akademischen Disziplin.

Eine kurze Studie zum aktuellen Thema Umweltzerstörung, Fortschritt und Entwicklung legt Manfred Wöhlcke in seinem Buch "Der Fall Lateinamerika - Die Kosten des Fortschritts" vor. Der Verfasser stellt interne Ursachen für Unterentwicklung in den Mittelpunkt seiner kurzen Einführung in die allgemeine Entwicklungsdiskussion. Für Lateinamerika faßt er diese unter dem Begriff des "Morbus Latinus" zusammen. Das Bild, das er in den folgenden Kapiteln zu den Themen "Soziale Entwicklung" und "Ökologische Entwicklung" zeichnet, kann man grob als die Perspektive des "Morbus Germanicus" charakterisieren: 
Eine Sichtweise, die den technischen und wirtschaftlichen Fortschritt an sich für Negatives verantwortlich macht und Fehlentwicklungen zum zentralen Begriff "Katastrophen" hochstilisiert (z.B. auf Seite 9, 22, 116), gleichzeitig aber auch alles Vergangene - einschließlich der europäischen Industrialisierung des 19. Jahrhunderts - romantisch verklärt (S. $16 \mathrm{f}$,, 118).

Viele Thesen des Buches werden auf allgemeine Zustimmung stoßen, bei anderen ist jedoch Widerspruch angebracht. So zum Beispiel, wenn für Lateinamerika diagnostiziert wird: "Die Menschen stehen im Dienst der Wirtschaft und nicht umgekehrt" (S. 23). Das Problem ist doch, daß viel zu viele nicht im Dienst der Wirtschaft stehen, sondern marginalisiert sind. Die sehr europäische Sichtweise schimmert etwa durch auf Seite 99, wenn das "Mißverhältnis zwischen einer prekären Grundausstattung und (weit verbreiteten) gehobenen Konsumgütem" bei den Wohnbedingungen hervorgehoben wird, wobei zu letzteren Radio, Fernsehgerät, Kühlschrank und Auto gezählt werden. Hier fällt zweierlei auf: Erstens, daß unsere Vorstellungen von dem, was "Grundausstattung" und was "gehobener Konsum" ist, unreflektiert übertragen werden. Zweitens aber auch, daß die Versorgung mit der "Grundausstattung" in den Ländern der Dritten Welt (ganz im Gegensatz zum sich entwickelnden Europa des 19. Jahrhunderts) samt und sonders vom Staat übernommen wird mit dem vom Autor beklagten Ergebnis, während die bessere Versorgung mit sogenannten "gehobenen Konsumgütern" gerade diejenigen Güter betrifft, für die sich der Staat auch in der Dritten Welt nicht einmischt.

Wer wissen will, daß es um Lateinamerika schlimm steht, wird in dem Buch eine knappe, aber sehr kenntnisreiche und dichte Argumentation finden; insbesondere zu Umweltproblemen in Brasilien (Wöhlcke ist vor allem Brasilien-Experte) sind die Nachweise aus der intemationalen und brasilianischen Literatur wertvoll und weiterführend. 